\title{
Production of Heavy Elements During the Explosion of a Low-Mass Neutron Star in a Close Binary
}

\author{
I.V. Panov ${ }^{1,2 *}$, A.V. Yudin ${ }^{1,2}$ \\ ${ }^{1}$ National Research Center "Kurchatov Institute" - ITEP \\ ${ }^{2}$ National Research Center "Kurchatov Institute"
}

\begin{abstract}
The nucleosynthesis of heavy elements in the scenario for the evolution of a close binary of neutron stars differing greatly in mass is considered. In contrast to the scenario for the merger of two neutron stars of comparable masses considered repeatedly in the literature, the evolution of such a binary at the final stage consists in a rapid mass transfer to the more massive star and an explosive disruption of the low-mass component. We provide the details of the explosion and calculate the abundances of the heavy elements produced in this process for various initial conditions.
\end{abstract}

Keywords: neutron stars, close binaries, nucleosynthesis, nuclear reactions, beta decay.

* email: panov_iv@itep.ru 


\section{INTRODUCTION}

The nucleosynthesis maintained by a rapid neutron capture (the r-process) is responsible for the production of more than half of all elements heavier than iron in nature. It results from the capture of neutrons and the subsequent beta decays of forming short-lived neutron-rich nuclei in an environment with a high neutron number density. The region where it proceeds on the map of nuclei lies near the neutron stability boundary (Burbidge et al. 1957; Cameron et al. 1957; Seeger et al. 1965).

A high initial neutron number density, up to 150 per seed nucleus (as a rule, such are the iron-peak nuclei), is needed to create the conditions for the r-process capable of producing heavy elements up to the heaviest ones. Such conditions are achieved in astrophysical objects in scenarios with a large neutron excess and a high density of matter, for example, during the merger of compact stellar remnants in close binaries or during the explosions of supernovae of a fairly rare type that form jets with a high neutron number density (Thielemann et al. 2017; Cowan et al. 2020) and in a hot wind from young neutron stars (Cameron 2001; Arcones and Thielemann 2013).

The first detection of a neutron star (NS) merger (Abbot et al. 2017) and the simultaneous observation of r-elements (Tanvir et al. 2017) confirmed the understanding that the main scenario for the r-process development is more likely associated with the ejecta that form during a NS merger at the end of the evolution of a close binary and not with supernova explosions (Hudepohl et al. 2010).

The details of the NS merger scenario have long been known (Freiburghaus et al. 1999) and the conditions for the synthesis of heavy elements in the r-process have been determined on their basis. The NSs forming a close binary approach each other due to the loss of angular momentum by the binary through the radiation of gravitational waves. At the final stage the stars merge into a single object - a supermassive NS or a black hole, with part of the matter being ejected from the binary in the form of jets or a wind. This is the so-called merging scenario most popular at present.

However, there exists another possibility: if the binary was initially highly asymmetric, then the stripping scenario can be realized (see Clark and Eardley 1977). In this scenario the more massive and compact star "devours" its less massive and more extended companion. Losing its mass in the course of such evolution, the latter approaches the in- 
nermost stable configuration of a minimum-mass NS and explodes. Blinnikov et al. (1984) first pointed to the connection of this process with gamma-ray bursts (GRBs).

In this paper we present the first nucleosynthesis calculation in the stripping scenario. The paper is structured as follows. In the next section we will briefly describe the stripping scenario. Then, we will consider the nucleosynthesis computation algorithm. Next, we will describe the dynamics of the explosive disruption of a low-mass NS. In the final section we will present the results of our r-process calculations and formulate our conclusions.

\section{STRIPPING MODEL}

The final evolutionary stages of a NS binary attract the increased attention of researchers. However, in almost all of the multidimensional hydrodynamic simulations performed so far the NS masses were close and fairly large, $M \gtrsim M_{\odot}$, and the result of their interaction was a merger (Korobkin et al. 2012; Rosswog et al. 2014; Martin et al. 2015). Indeed, the radius of such NSs depends weakly on the mass (Lattimer and Prakash 2001) and they behave like two liquid droplets when in contact, merging into a single object - a supermassive NS or a black hole.

However, if the binary is highly asymmetric, i.e., the component masses differ significantly and, moreover, the low-mass NS mass is sufficiently small, then the stripping scenario can be realized (Clark and Eardley 1977). As the binary components approach each other, the lower-mass NS is the first to overfill its Roche lobe and begins to flow onto the more massive companion. During such a mass transfer it can reach the lower NS mass limit (0.1 $M_{\odot}$; see, e.g. Haensel et al. 2007) and explode, actually producing a GRB (Blinnikov et al. 1984, 1990).

The interest in the stripping model was renewed after the historic identification of the gravitational signal GW170817 and GRB170817A (Abbot et al. 2017). Observations showed that many parameters of this GRB, which turned out to be very peculiar, are close to the predictions of the stripping model (for a discussion, see Yudin et al. 2019).

The results of our hydrodynamic simulations of the explosive disruption of a minimum-

mass NS (see also Blinnikov et al. 1990; Sumiyoshi et al. 1998) were used to determine the pattern of nucleosynthesis in this case. 
Under conditions of a high neutron number density the nucleosynthesis in the r-process proceeds near the neutron stability boundary, which requires predicting all characteristics of short-lived experimentally unstudied nuclei. An additional complexity in simulating the r-process in a highly neutron-rich environment, which is also typical for a NS merger, is fission. As was first shown in numerical simulations of the r-process in the NS merger scenario (see, e.g., Freiburghaus et al. 1999), the fission of heavy-element nuclei leads to nucleosynthesis cycling (Panov et al. 2003), i.e., to the drawing of a large number of fission product nuclei as new seed nuclei into the r-process and to the production of most heavy elements from the second peak in the elemental abundance curve to thorium and uranium. The corresponding increase of the theoretical data used in the simulations, such as the rates of induced, delayed, and spontaneous fission as well as the mass distribution of fission product nuclei, and their inclusion as new seed nuclei (Panov et al. 2003, 2008, 2009) makes the system of equations and the simulation process more complicated and requires optimizing the numerical schemes and algorithms.

For our numerical simulations of the r-process we applied the nuclear reaction network previously implemented in the SYNTHeZ code (Blinnikov and Panov 1996; Nadyozhin et al. 1998), which specifies the number densities of all the nuclei drawn into the nucleosynthesis, including the neutron number density control. In the updated SYNTHeR (nucleoSYNThesis of HEavy elements in the R-process) code (Korneev and Panov 2011) the fission reactions were supplemented by a proper allowance for the mass distribution of fission product nuclei and their return to the r-process as new seed nuclei, which leads to the establishment of a quasi-steady current of nuclei.

Since the nucleosynthesis is studied in the scenarios of both explosive nucleosynthesis at high temperatures and densities and the transition from the explosive synthesis of elements to the alpha-process and the r-process, the nucleosynthesis codes were supplemented by the reactions with charged particles and the previously disregarded interaction of nucleons and nuclei with electrons (Panov et al. 2018). We supplemented the SYNTHeR code by the weak interaction reactions, whose bank (Langanke and Martinez-Pinedo 2000) contains data for the isotopes of the iron-peak elements $(20<Z<32)$. There are no lighter elements $(Z<20)$ in this bank, although, as has been recently shown (Fischer et al. 2016), the role of light elements is also quite significant and, therefore, their reactions with electrons should also be taken into account. The weak processes are especially important at high temperatures $\left(T>5 \times 10^{9} \mathrm{~K}\right)$ and densities $\left(\rho>10^{8} \mathrm{~g} / \mathrm{cm}^{3}\right)$ and lead, in particular, 
to a change in the electron fractions $Y_{\mathrm{e}}$.

Since the reaction rates of the listed processes determining the eigenvalues of the Jacobi matrix for the system of differential equations realized in our nucleosynthesis codes differ in absolute value by orders of magnitude, the system of nucleosynthesis equations is a classic example of a stiff system of ordinary differential equations (ODEs). Many methods have been developed for the numerical integration of stiff systems of ODEs; the method by Gear (1971) was recognized as one of the most efficient ones.

The predictor-corrector method with the automatic choice of a step and an order of the method underlies the algorithm. The main difficulty in implementing this algorithm is the necessity of solving a very large system of linear equations (of the order of several thousand-by the number of equations in the nucleosynthesis system) when performing the corrector iterations. Since the matrix of coefficients in this system is sparse, special methods developed for sparse matrices (see, e.g., Pissanetzky 1984), in particular, the special software package for astrophysical problems (Blinnikov et al. 1993), which allowed some algorithms to be accelerated by 1-2 orders of magnitude, were applied for its solution. Note that the choice of a method for solving a sparse system has a decisive influence on the efficiency of the entire algorithm for the kinetic problem.

The popular methods (Gibbs et al. 1976) suggesting that the matrix has a symmetric structure are not always a good approximation for a real problem (Lyutostanskii et al. 1986). Therefore, we chose the algorithm for sparse matrices with an arbitrary structure (Osterby and Zlatev 1983) implemented in both SYNTHeZ and SYNTHeR codes that have an internal check for the conservation

The boundaries of the region of the nuclides involved in the nucleosynthesis were defined as $Z_{\min }=1$ and $Z_{\max }=110$, while $A_{\min }$ and $A_{\max }$ were specified according to the mass model used: the extended Thomas-Fermi model with the Strutinsky integral (Aboussir et al. 1995) or the liquid-drop model (Moeller et al. 1995). Thus, the total number of nuclei $\mathrm{N}$ involved in the nucleosynthesis was determined.

The nuclear reaction rates, which are the coefficients in the differential equations, were calculated for the same mass models. All pair and other major burning reactions, along with the alpha-decay and fission reactions and weak interactions, enter into the list of involved nuclear reactions. They include: all pair reactions with neutrons, protons, 
alpha particles, and gamma-ray photons; the beta-decay and beta-delayed processes, such as the emission of several neutrons during beta decay and delayed fission; the induced and spontaneous fission; a number of other important reactions, such as the $3-\alpha$ reaction and the ${ }^{12} \mathrm{C},{ }^{16} \mathrm{O}$ and ${ }^{28} \mathrm{Si}$ burning reactions.

The applied scheme allows the nucleosynthesis to be efficiently calculated in various scenarios at $T_{9}<7$ and $\rho<10^{12} \mathrm{~g} / \mathrm{cm}^{3}$. Our main calculations were made with the widely used rates of beta decay and delayed neutrons (Moeller et al. 1997, 2003), alpha decay (Moeller et al. 2003), the rates of thermonuclear reactions (Rauscher and Thielemann 2000) and fission (Panov et al. 2005, 2010, 2013; Korneev and Panov 2011). The experimentally measured beta decay rates were taken from the NuDat2 nuclear database (2009). The neutron-capture rates by heavy nuclei (for elements with $Z>83$ ) and the neutron-induced fission rates are based on the calculations by Panov et al. (2010), while the delayed fission rates were taken from Panov et al. $(2005,2010)$.

\section{EXPLOSION OF A LOW-MASS NS}

A minimum-mass NS has a peculiar structure. Figure 1 shows the dependence of the logarithm of the density $\lg \rho$ on radial coordinate $r$ calculated using the equation of state from Haensel and Potekhin (2004). The corresponding Lagrangian (mass) coordinates $m$ are shown on the upper axis. The structure of the matter is clearly seen: the NS core composed of nuclear matter lies at the center. The core is surrounded by a mantle: a layer of exotic nuclear configurations ("lasagne", "pasta" etc.; see Haensel et al. 2007) and hyper-nuclei immersed into a sea of free neutrons. This part, containing $90 \%$ of the mass of the entire star, has a radius slightly greater than $10 \mathrm{~km}$.

The outer crust consists of a sequence of mono-layers of nuclei (Ruster et al. 2006), from highly neutron-rich isotopes, such as ${ }^{118} \mathrm{Se}$, to ${ }^{56} \mathrm{Fe}$ on the surface. The specific sequence of nuclei can slightly change, depending on the mass formula used and other parameters of the equation of state for the NS crust (see, e.g., Martin et al. 2015), but the general trend remains the same.

The symbols of different shapes in the figure indicate the initial data for the four trajectories used to calculate the nucleosynthesis. Some of their parameters are given in Table 1.

Consider the explosive disruption of a minimum mass NS by assuming the problem 


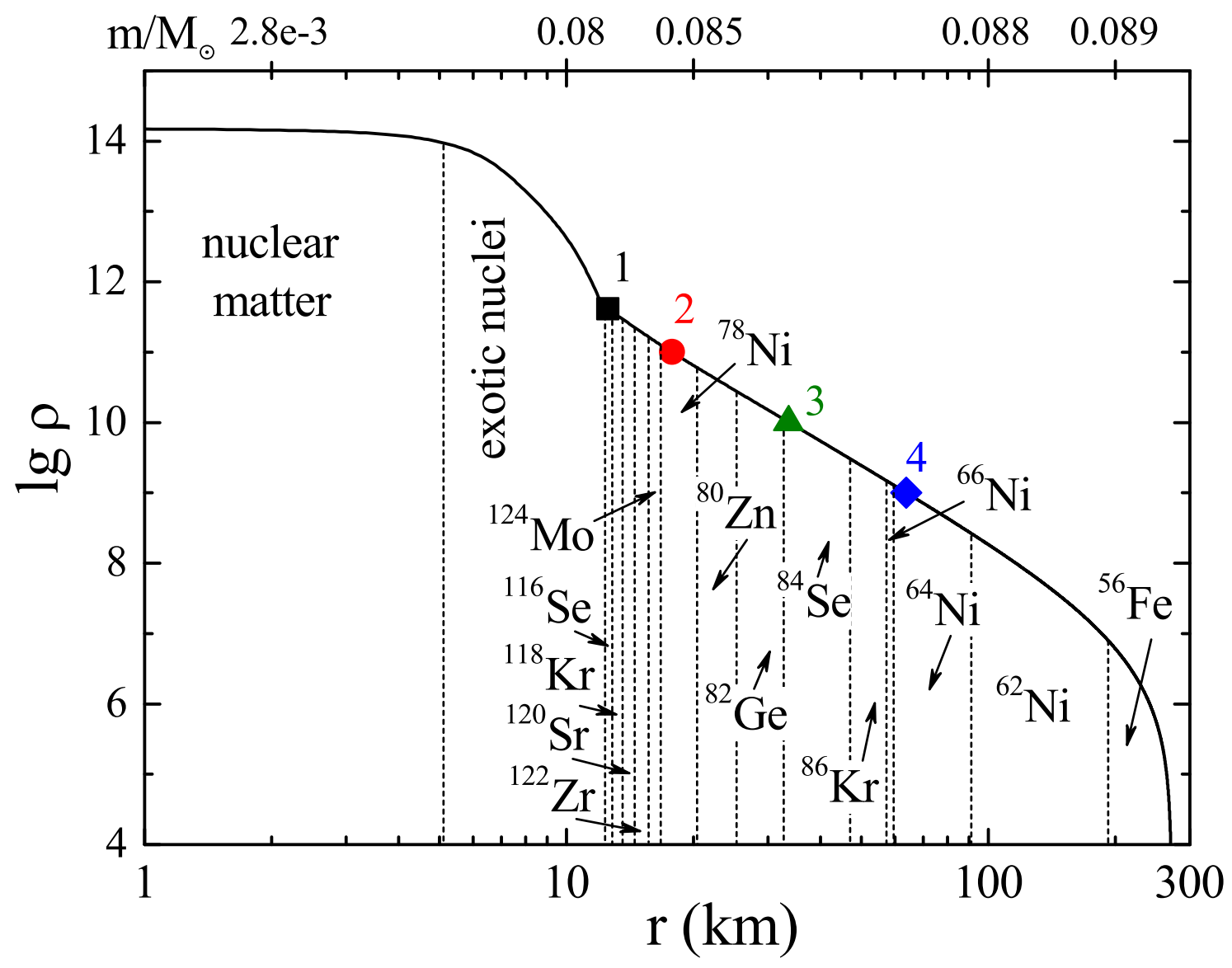

Fig. 1: Structure of a minimum-mass NS. The symbols mark the initial positions of the four trajectories used to calculate the nucleosynthesis.

to be spherically symmetric (see Yudin et al. 2019). The dynamics of this process was first computed by Blinnikov et al. (1990). Figure 2 shows the expansion velocity profiles for the NS matter as a function of mass coordinate $m$. The numbers mark the time (in seconds) from the loss of hydrodynamic stability by the star. The expansion begins from the stellar surface and the rarefaction wave reaches the center by $t_{5}=0.371$. By this time almost the entire star already expands with a speed $\sim 0.1$ of the light speed and the acceleration wave travels in the opposite direction, outward. In this case, an important event occurs: approximately between $t_{6}=0.373$ and $t_{7}=0.375$ this wave crosses the boundary of the NS mantle-crust at $m \approx 0.08 M_{\odot}$. While accelerating along a sharply falling density profile (Fig. 1), it turns into a shock wave and heats the matter.

The behavior of the density and temperature on the four chosen trajectories (see Table 1 and Fig. 1) is shown in Fig. 3. Initially, the density and temperature drop due to the overall expansion. Subsequently, at $t \approx 0.365$, the matter begins to be heated 
Table 1. Parameters of the trajectories used to calculate the nucleosynthesis

\begin{tabular}{|c|c|c|c|c|c|}
\hline \hline $\begin{array}{c}\text { Variant, } \\
\text { No }\end{array}$ & $\begin{array}{c}\text { Initial } \\
\text { composition }\end{array}$ & $T_{9}^{\max }$ & $\begin{array}{c}\rho_{0}^{\max }, \\
\mathrm{g} / \mathrm{cm}^{3}\end{array}$ & $\begin{array}{c}r_{0}, \\
\mathrm{~km}\end{array}$ & $Y_{\mathrm{e}}$ \\
\hline 1 & ${ }^{116} \mathrm{Se}$ & 0.93 & $4 \times 10^{11}$ & 12.5 & 0.25 \\
\hline 2 & ${ }^{78} \mathrm{Ni}$ & 2.5 & $10^{11}$ & 17.8 & 0.335 \\
\hline 3 & ${ }^{84} \mathrm{Se}$ & 6.3 & $10^{10}$ & 33.8 & 0.405 \\
\hline 4 & ${ }^{64} \mathrm{Ni}$ & 10 & $10^{9}$ & 63.5 & 0.44 \\
\hline
\end{tabular}

by the acoustic oscillations inevitably generated as the central part of the star expands. Propagating away from the core along a falling density profile, accelerating, and turning into weak shocks, these small perturbations heat the matter. Later, already by $t \approx 0.375$, a strong shock approaches the stellar layers under consideration (its formation is seen in Fig. 2 between the profiles marked by 7 and 8 ) and causes a very fast increase in density (by a factor of 2-3) and temperature (by orders of magnitude). This time is especially difficult for the nucleosynthesis calculations primarily because the thermonuclear reaction rates change abruptly and the parameters of the numerical algorithm are violated, which can lead to a numerical instability of the algorithm for solving the equations. After reaching the peak values, the density and temperature continue to drop. As our hydrodynamic calculations show, they decrease due to the expansion of the matter in a regime faster than its free expansion: $\rho \sim t^{-3.6}, T \sim \rho^{2 / 3} \sim t^{-2.4}$

It is easy to estimate the parameters of the NS binary considered by us at the explosion time. The Roche lobe radius $R_{\mathrm{R}}$ for the low-mass NS approximately coincides with its radius, i.e., $R_{\mathrm{R}} \approx R_{\mathrm{s}} \approx 270 \mathrm{~km}$ (Fig. 1). The separation $a$ between the binary components is related to the Roche lobe size by an approximate relation (Paczynski 1971):

$$
\frac{R_{\mathrm{R}}}{a} \approx 0.462\left(\frac{q}{1+q}\right)^{1 / 3}
$$

where $q=m_{2} / m_{1}$ is the component mass ratio. Taking $m_{1}=1.4 M_{\odot}$ and $m_{2}=0.1 M_{\odot}$ for our estimate, we will get $a \approx 1441 \mathrm{~km}$. The escape speed from the surface of the low-mass NS is $V_{\mathrm{esc}}=\sqrt{\frac{2 G m_{2}}{R_{\mathrm{s}}}} \approx 10^{9} \mathrm{~cm} / \mathrm{sec}$; the escape speed from the field of the massive component is $V_{\mathrm{esc}}=\sqrt{\frac{2 G m_{1}}{a}} \approx 1.6 \times 10^{9} \mathrm{~cm} / \mathrm{sec}$ Thus, the matter ejected during the low-mass NS explosion has speeds (Fig. 2) that exceed the escape speed at least by several times. In reality, in these simples estimates we disregarded the orbital motion of the components, but detailed numerical simulations (Manukovskiy 2010) of the expansion of the matter in the NS binary under consideration confirm our conclusion. 


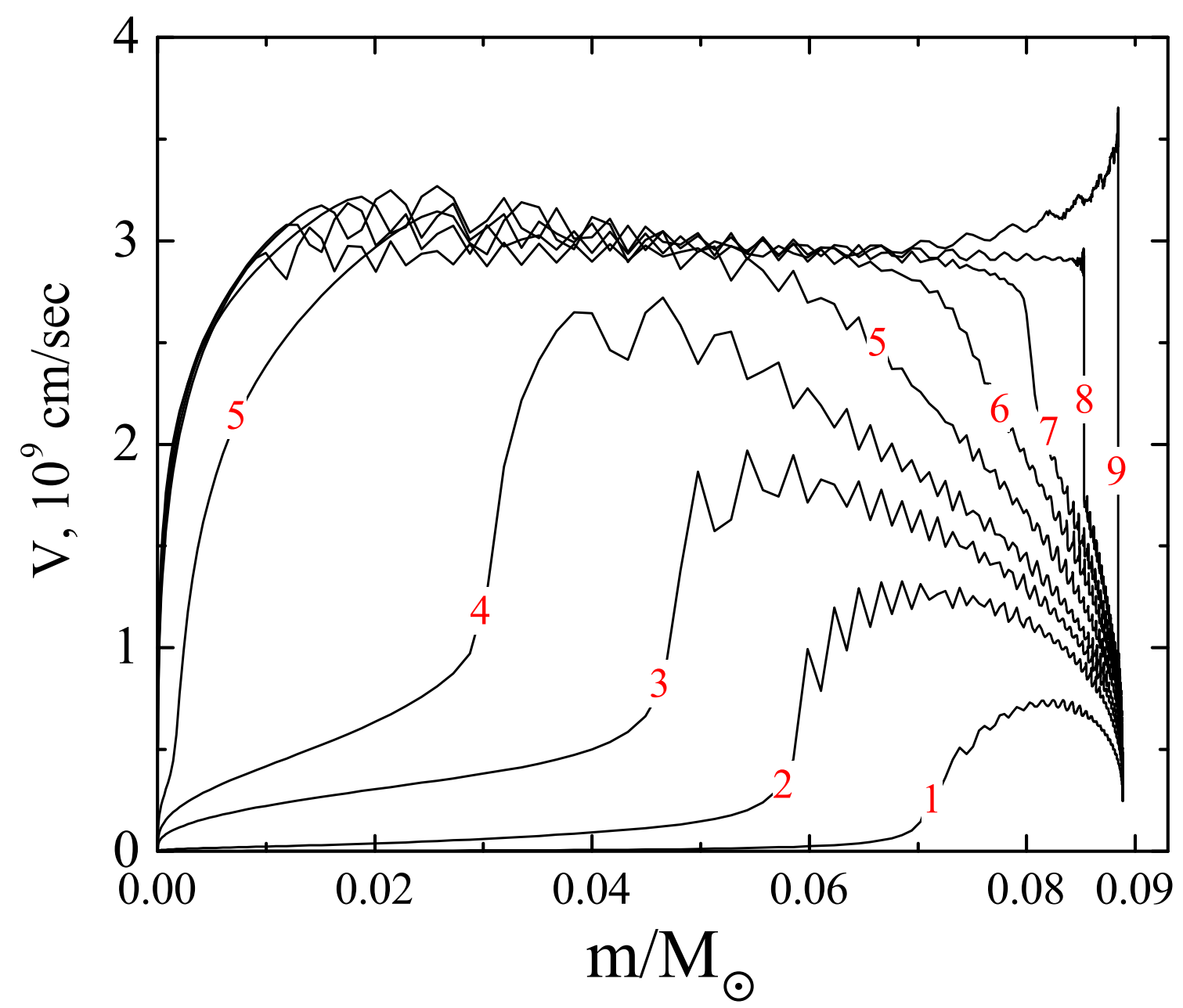

Fig. 2: Expansion velocity profiles for the NS matter as a function of mass coordinate $m$. The numbers mark the times (in seconds): $t_{1}=0.360, t_{2}=0.365, t_{3}=0.368$, $t_{4}=0.369, t_{5}=0.371, t_{6}=0.373, t_{7}=0.375, t_{8}=0.376, t_{9}=0.378$.

\section{PRODUCTION OF HEAVY ELEMENTS DURING A LOW-MASS NS EXPLOSION}

We calculated the nucleosynthesis of heavy elements along the above-described most typical trajectories in the off-line mode. We determined the evolution of the composition at a temperature $T_{9}>7$ in the NSE approximation (NSE stands for nuclear statistical equilibrium) and used the SYNTHeR code (Korneev and Panov 2011) to calculate the elemental abundances as the temperature decreased. The transition regime, when the over-compressed matter at a subnuclear density whose composition is defined by the equation of state rapidly passes to the qualitatively different state of a dense hot plasma described, in particular, by the Boltzmann-Maxwell equations and the kinetic model of nucleosynthesis developed for such conditions (Blinnikov and Panov 1996), is most difficult 


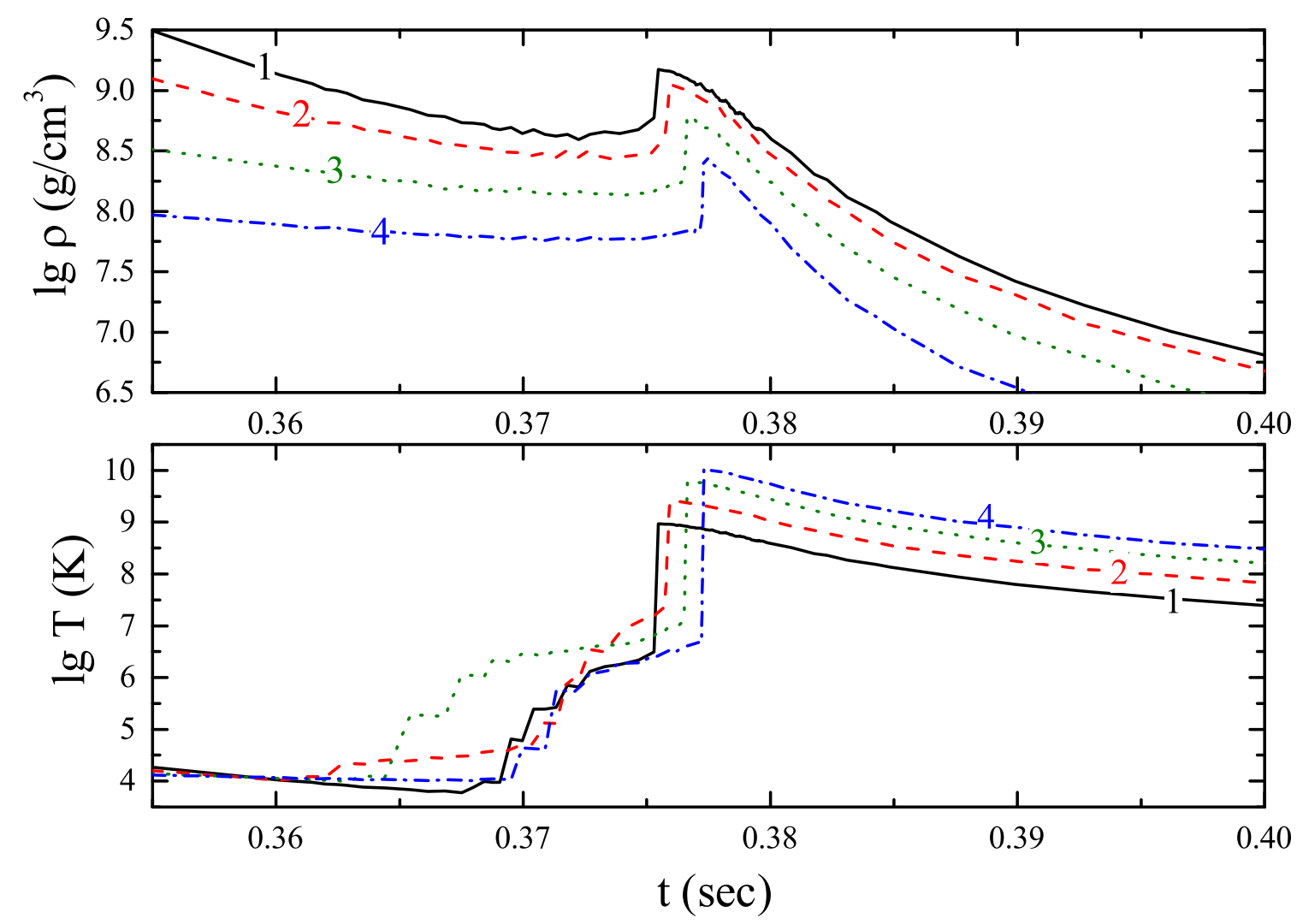

Fig. 3: Evolution of the density and temperature along the four trajectories 1-4 considered as a function of time.

for the simulations. Therefore, the transition from the subnuclear matter composed of exotic neutron-overrich nuclei to the nuclear reaction network was made formally. The basis formaking this transition was the conservation of the preexplosion local electron fraction $Y_{\mathrm{e}}$ (which determines the degree of matter neutronization) up to the onset of nucleosynthesis. As the model is developed further, this problem will be solved iteratively in the nucleosynthesis calculations in combination with the expansion hydrodynamics of the matter both by tracing its local heating by the shock and by taking into account the energy release during beta decay and fission.

Below we discuss the results of our nucleosynthesis calculations for four trajectories characterized by different initial $Y_{\mathrm{e}}$ and initial isotopic compositions (see Table 1). Figure 4 shows the evolution of the electron fraction, with the increase in $Y_{\mathrm{e}}$ (at its initial values less than 0.4) implicitly reflecting the intensity and duration of the r-process begun after the second expansion wave $t \approx 0.37$. The electron fraction $Y_{\mathrm{e}}$ changes most dramatically due to the beta decays both along the path of the r-process and at the cooling stage as a result 
of the decay of unstable isotopes and the production of stable elements only if $Y_{\mathrm{e}} \leq 0.4$. The role of thermonuclear reactions in heating the matter during the shock passage for variants 1 and 2 is relatively minor (variant 2) or small (variant 1) and the electron-toproton ratio changes monotonically as the beta decays of the neutron-rich nuclei produced predominantly in the r-process occur. $Y_{\mathrm{e}}$ in nucleosynthesis increases along trajectory 1 considerably longer than along trajectory 2 due to the beta decay of a large number of long-lived isotopes of rare-earth and trans-uranium elements. Under strong heating (which is especially clearly seen for variant 4) the role of thermonuclear reactions in the matter during the shock passage increases, but the numerical effect related to the rapidly varying processes at the shock front can also have a noticeable influence on the jump in $Y_{\mathrm{e}}$. This aspect of the problem requires additional studies.

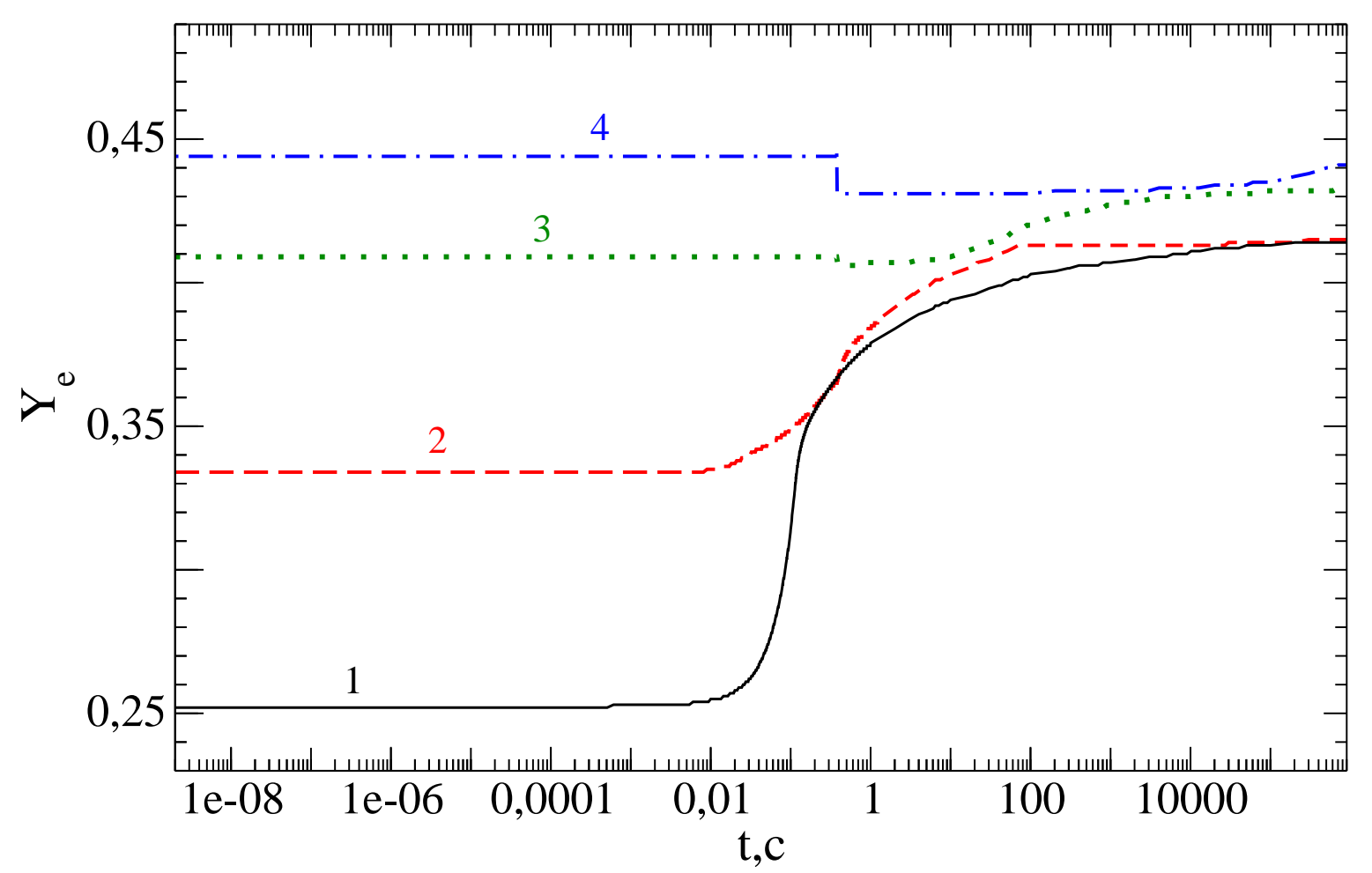

Fig. 4: Electron fraction $Y_{\mathrm{e}}$ versus time on the four chosen trajectories. The numbers near the curves are the trajectory numbers.

Figure 5 shows the evolution of the number density of free neutrons as the r-process develops. It follows from the figure that a level of free neutrons $N_{n} \geq 10^{22} \mathrm{~cm}^{-3}$ sufficient for the r-process to proceed is maintained for several hundred milliseconds, which is enough to produce all of the heavy nuclei up to uranium only along trajectories 1 and 2 (Fig. 6). 
Accordingly, for variants 3 and 4, for which the initial neutron excess decreases very rapidly during the transition from a subnuclear density to densities of the order of the hot-wind densities, the r-process does not proceed and new elements are produced predominantly through the $(\alpha, X)$-reactions, including those produced during the matter heating by the shock and the burst of nucleosynthesis due to the acceleration of thermonuclear reactions.

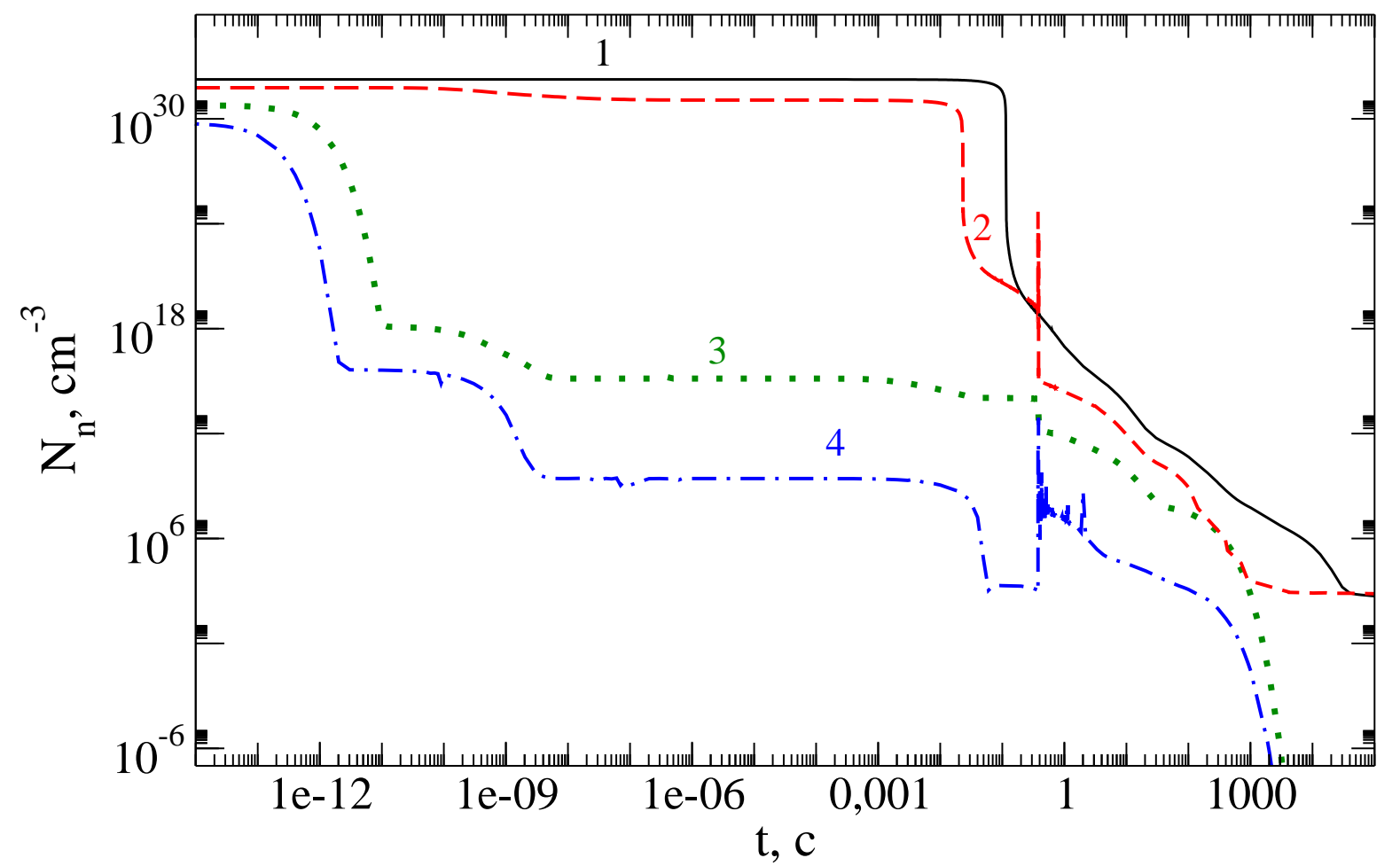

Fig. 5: Number density of free neutrons $N_{n}$ versus time on the four chosen trajectories. The numbers near the curves are the trajectory numbers.

The jumps in $N_{\mathrm{n}}$ (variants 2 and 4 ) seen in Fig. 5 at $t \sim 0.37$ or the sharp absorption of neutrons (in variant 3) occur with the switch-on of thermonuclear reactions during the shock passage and are largely attributable to the numerical effects whose influence on the solution is minor. Note that the significant and gradual decrease in $N_{n}$ at short times $t<10^{-12}, 10^{-8}, 0.01 \mathrm{~s}$ is determined mainly by the switch-on of charge-exchange nuclear reactions.

The peaks in the region of atomic masses with $A \sim 130$ and 196 for variants 1 and 2 (Fig. 6) are well-structured and are consistent with the observations in magnitude and place. The rare-earth peak $A \sim 160-170$ for variant 1 is formed, which is even excessive compared to the observational data. 


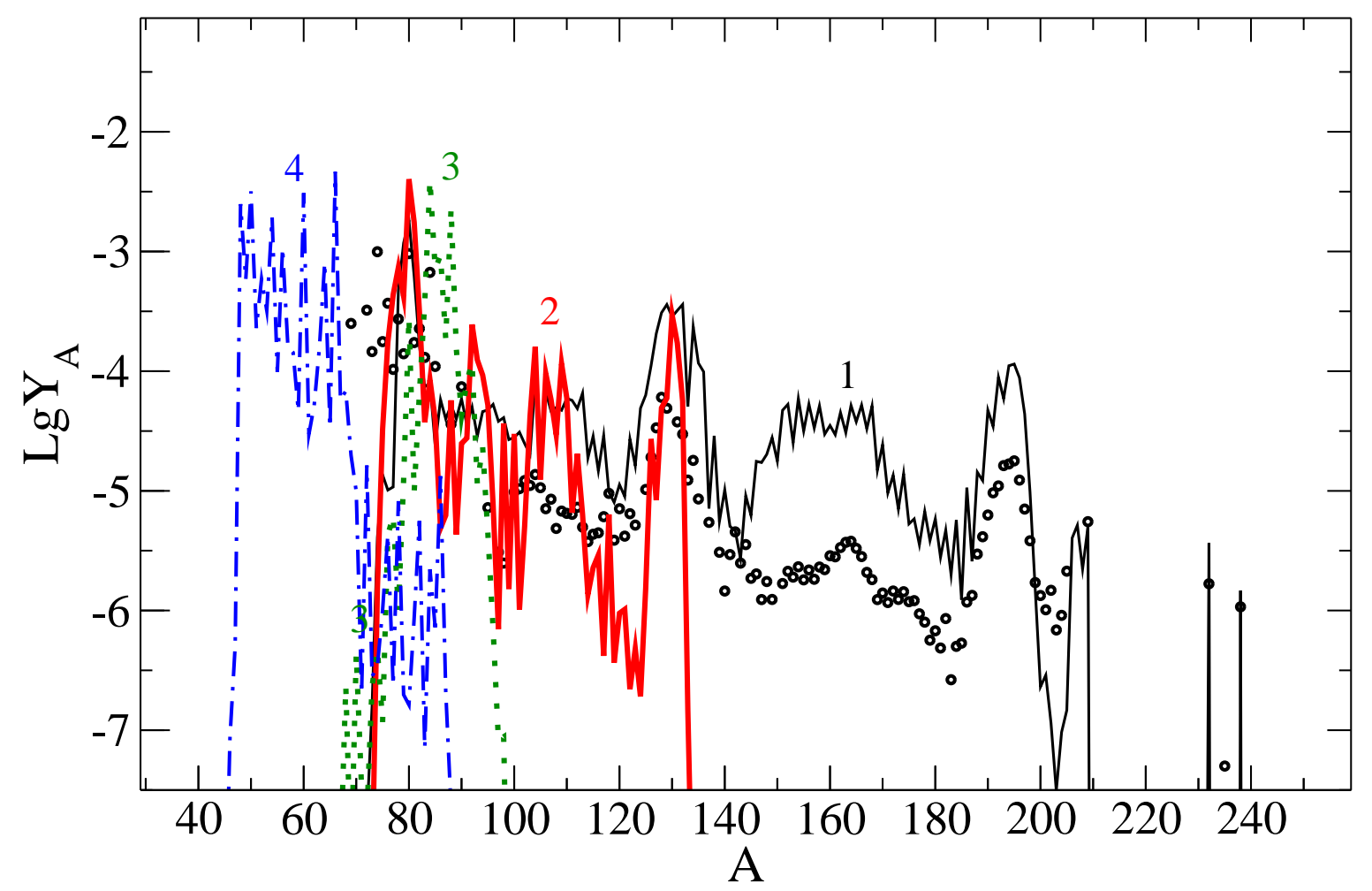

Fig. 6: Abundances of the chemical elements produced at the end of nucleosynthesis along the four chosen trajectories. The dots indicate the abundances of the heavy elements produced in the Solar System. The numbers near the curves are the trajectory numbers.

\section{CONCLUSIONS}

The evolution of close binaries can be different: under some conditions a NS merger occurs; under other ones a mass transfer between the companions (stripping) is possible, which ends with a low-mass NS explosion. The masses of the binary components must differ greatly for the stripping mechanism to be realized.

The fraction of binary stars with a low-mass companion among the entire population of binary NSs is apparently small. Such a binary configuration, whose formation probability is yet to be determined, will represent a fraction of the stripping mechanism of GRBs in their total population.

The original results obtained from our nucleosynthesis calculations in the scenario for the evolution of two NSs with significantly different masses show that in the stripping scenario during the evolution of two NSs part of the crust and mantle matter is neutronized strongly enough for the r-process to proceed in it during the explosion and expansion with 
the production of a large amount of heavy elements. The abundance curve of the heavy nuclei $Y(A)$ produced during low-mass NS disruption, on the whole, agrees well with both heavy-element abundance observations and heavy-element abundance calculations for a classical NS merger. For some trajectories the heavy-element abundance combines the abundance of the "heavy" fraction of the elemental abundance typical for the ejection in the NS merger scenario and the "light" component forming in the winds from a hot massive neutron remnant in the same NS merger scenario. It follows from Fig. 6 that when the third peak is formed (variant 1), the "iron" peak of elements with mass numbers $\sim 80$ does not burn out, which differs from the heavy-element production dynamics in the NS merger scenario, where the second and third peaks are formed (the main r-process) in the jets or only the first and second peaks are formed (an incomplete r-process in the wind).

The nucleosynthesis was calculated offline on fixed tracks, without integrating the nucleosynthesis contribution for all the possible trajectories, which allows the difference between the nucleosynthesis calculations for different layers of ejected matter to be estimated. In fact, this is the first step in the project of research on the stripping scenario and the nucleosynthesis proceeding during the explosion of a low-mass remnant. Our final goal is a self-consistent calculation of the low-mass NS explosion that takes into account, among other things, the additional heating of the matter during intense nucleosynthesis due to beta decay and fission. We are working on this problem at present.

\section{ACKNOWLEDGMENTS}

We are grateful to S.I. Blinnikov and D.K. Nadyozhin for the discussion of physical processes at the final evolutionary stages of a NS binary and the equation of state for superdense matter and to N.I. Kramarev for his participation in the discussion and his interest in the work. This work was supported by the Russian Foundation for Basic Research (project no. 18-29-21019 mk). 


\section{REFERENCES}

1. B.P. Abbot, et al., Astrophys. J. Lett. 848, L12, L13, (2017).

2. Y. Aboussir, J. M. Pearson, A. K. Dutta, and F. Tondeur, At. Data Nucl. Data Tables bf 61, 127 (1995).

3. A. Arcones and F-K. Thielemann, J. Phys. G: Nucl. Part. Phys. 40, 013201 (2013).

4. S.I. Blinnikov, I.D. Novikov, T.V. Perevodchikova, A.G. Polnarev, Sov. Astron. Lett. 10, $177(1984)$.

5. S.I. Blinnikov, V.S. Imshennik, D.K. Nadyozhin, I.D. Novikov, T.V. Perevodchikova, and A.G. Polnarev, Sov. Astron. 34, 595 (1990).

6. S.I. Blinnikov and O.S. Bartunov, Astron. Astrophys. 273, 106 (1993).

7. S.I. Blinnikov and I.V. Panov, Astron. Lett. 22, 39 (1996).

8. E.M. Burbidge, G.R. Burbidge, W.A. Fowler, and F. Hoyle, Rev. Mod. Phys. 29, $547(1957)$.

9. N.E. Gibbs, W.G. Poole, and P.K. Stockmeyer, SIAM J. Numer. Anal. 13, 236 (1976).

10. C.W. Gear, Numerical Initial Value Problems in Ordinary Differential Equations (Prentice-Hall, Englewood Cliffs, NJ, 1971).

11. J.P.A .Clark and D.M. Eardley, Astrophys. J. 215, 311 (1977).

12. O. Korobkin, S. Rosswog, A. Arcones, and C. Winteler, MNRAS 426, 1940 (2012).

13. I.Yu. Korneev, I.V. Panov, Astron. Lett. 37, 864 (2011).

14. J.J. Cowan, C. Sneden, T.C. Beers, J.E. Lawler, A. Aprahamian, M. Wiescher, K. Langanke, G. Martinez-Pinedo, and F-K. Thielemann, arXiv:1901.01410 [astro-ph.HE], submitted to Reviews of Modern Physics (2020).

15. A.G.W. Cameron, Publ. Astron. Soc. Pac. 69, 201 (1957).

16. A.G.W. Cameron, Astroph. Journal, 562, 456 (2001).

17. K. Langanke, G.Martinez-Pinedo, Nuclear Physics A 673, 481 (2000).

18. Lattimer J.M., Prakash M., Astrophys. J., 550, 426 (2001)

19. Yu.S. Lyutostanskii, I.V. Panov, O.N. Sinyukova, S.S. Filippov, and V.M. Chechetkin, Sov. J. Nucl. Phys. 4, 47 (1986). 
20. K.V. Manukovskiy, Astron. Lett. 36 3, 191 (2010).

21. D. Martin, A. Perego, A. Arcones, F.-K. Thielemann, O. Korobkin, and S. Rosswog, Astrophys.1 J. 813, 2 (2015).

22. P. Moeller, J. R. Nix, W. D. Myers, W. J. Swiatecki, ADNDT 59, 185 (1995).

23. P. Moeller, J.R. Nix, and K.-L. Kratz, Atomic Data Nucl. Data Tables 66, 131 (1997).

24. P. Moeller, B. Pfeiffer, and K.-L. Kratz, Phys. Rev. C 67, 055802 (2003).

25. D.K. Nadyozhin, I.V. Panov, and S.I. Blinniko), Astron. Astrophys. 335, 207 (1998).

26. I.V. Panov, F.-K. Thielemann, Astron. Lett. 29, 510 (2003).

27. I.V. Panov, E. Kolbe, B. Pfeiffer, T. Rauscher, K.-L. Kratz, and F.-K. Thielemann, Nucl. Phys. A 747, 633 (2005).

28. I.V. Panov, I.Yu. Korneev, T. Rauscher, and F.-K. Thielemann, Astron. Astrophys. 513, A61 (2010).

29. I.V. Panov, I.Yu. Korneev, F.-K. Thielemann, Astron. Lett. 34, 189 (2008).

30. I.V. Panov, I.Yu. Korneev and F.-K. Thielemann, Phys. Atomic Nucl. 72, 1026 (2009).

31. I.V. Panov, I.Yu. Korneev, Yu.S. Lutostansky, and F.-K. Thielemann, Phys. Atomic Nucl. 76, 88 (2013).

32. I.V. Panov, I.Yu. Korneev, G. Martinez-Pinedo, and F.-K. Thielemann, Astron. Lett. 39, 150 (2013).

33. I.V. Panov, S.I. Glazyrin, F. Roepke, S.I. Blinnikov, Astron. Lett. 44, 309 (2018).

34. B. Paczynski, Ann. Rev. Astron. Astrophys. 9, 183 (1971).

35. S. Pissanetzky, Sparse matrix Technology, Acad. Press, London Orlando etc., (1984)

36. T. Rauscher and F.-K. Thielemann, Atomic Data Nucl. Data Tabl. 5, 1 (2000).

37. S. Rosswog, O. Korobkin, A. Arcones, F.-K. Thielemann, and T. Piran, MNRAS 439, 744 (2014).

38. S.B. Ruster, M. Hempel, and J. Schaffner-Bielich, Phys. Rev. C 73, 3 (2006).

39. P.A. Seeger, W.A. Fowler, and D. Clayton, Astrophys. J. Suppl. Ser. 11, 121 (1965).

40. K. Sumiyoshi, S. Yamada, H. Suzuki, and W. Hillebrandt, Astron. Astrophys. 334, 159 (1998). 
41. N.R. Tanvir, A.J. Levan, C. Gonzalez-Fernandez, O. Korobkin et al), Astrophys. J., 848, L27 (2017).

42. F.-K. Thielemann, M. Eichler, I.V. Panov, and B. Wehmeyer, Annu. Rev. Nucl. Part. Sci. 67, 253 (2017).

43. T. Fischer, G. Martinez-Pinedo, M. Hempel, L. Huther, G. Ropke, S. Typel, and A. Lohs, EPJ Web Conf. 109, 06002 (2016).

44. C. Freiburghaus, S. Rosswog, and F-K. Thielemann, Astrophys. J. 525, L121D (1999).

45. P. Haensel and A.V. Potekhin, Astron. Astrophys. 428, 191 (2004).

46. P. Haensel, A.Yu. Potekhin, and D.G. Yakovlev, Neutron Stars, Equation of State and Structure (Springer, New York, NY, 2007), v. 1, p. 619.

47. L. Hudepohl, B. Mueller, H-T. Janka, A. Marek, A. Marek, and G.G. Raffelt, Phys. Rev. Lett. 104, 251101 (2010).

48. O. Osterby and Z. Zlatev, Direct Methods for Sparse Matrices (Lecture Notes in Computer Science. 157, (1983). Springer, Berlin-Heidelberg-New York-Tokyo)

49. A.V. Yudin, T.L. Razinkova, S.I. Blinnikov, Astron. Lett. 45, 847 (2019).

50. NuDat2, 2009, National Nuclear Data Center, Information Extracted from the NuDat 2 Database, http://www.nndc.bnl.gov/nudat2/ 